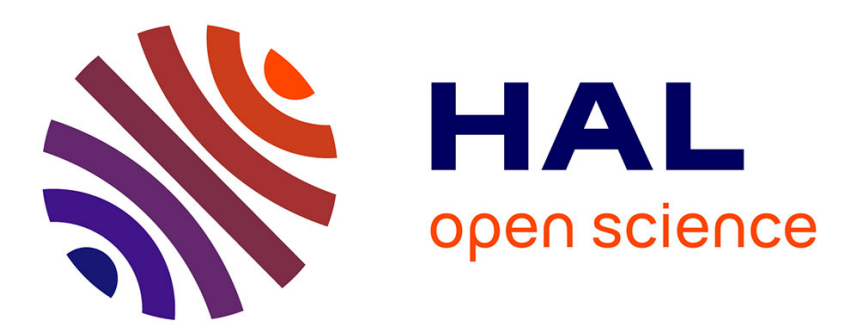

\title{
Le développement d'un index d'exposition de soi dans les médias sociaux : phase exploratoire d'identification des indicateurs constitutifs
}

Oliviane Brodin, Lise Magnier

\section{- To cite this version:}

Oliviane Brodin, Lise Magnier. Le développement d'un index d'exposition de soi dans les médias sociaux : phase exploratoire d'identification des indicateurs constitutifs. Revue management \& avenir, 2012, Analyse du comportement de l'internaute, 58 (8), pp. 144-168. 10.3917/mav.058.0144 . hal03350215

\section{HAL Id: hal-03350215 \\ https://hal-univ-artois.archives-ouvertes.fr/hal-03350215}

Submitted on 29 Sep 2021

HAL is a multi-disciplinary open access archive for the deposit and dissemination of scientific research documents, whether they are published or not. The documents may come from teaching and research institutions in France or abroad, or from public or private research centers.
L'archive ouverte pluridisciplinaire HAL, est destinée au dépôt et à la diffusion de documents scientifiques de niveau recherche, publiés ou non, émanant des établissements d'enseignement et de recherche français ou étrangers, des laboratoires publics ou privés. 


\title{
Le développement d'un index d'exposition de soi dans les médias sociaux : phase exploratoire d'identification des indicateurs constitutifs ${ }^{1}$
}

\author{
Par Oliviane Brodin ${ }^{2}$ et Lise Magnier ${ }^{3}$
}

\section{Résumé}

Cet article a pour objet de clarifier le concept d'exposition de soi dans les médias sociaux. Il présente la première étape du développement d'un index d'exposition de soi sur la base d'une revue de littérature enrichie par une étude qualitative exploratoire. Les résultats montrent que l'exposition de soi est composée de deux sources constitutives, l'extimité relationnelle empathique et la visibilité de comparaison sociale dont les indicateurs respectifs sont identifiés.

Mots-clés : Exposition de soi, médias sociaux, visibilité, « extimité »

\section{The development of an index of self-exposure in social media: exploratory stage for identifying the formative indicators}

\begin{abstract}
The aim of this article is to clarify the concept of self-exposure in social media. Based on a literature review enriched with a qualitative exploratory research, this study presents the first stage in the development of an index of self-exposure along with the identification of its formative indicators. Results show that self-exposure is composed of two constitutive sources, (i) empathic and relational extimacy and (ii) social comparative visibility. Their respective indicators are identified.

Key words: Self-exposure, social media, visibility, "extimacy"

\footnotetext{
${ }^{1}$ Une présentation des résultats de cette étude a fait l'objet d'une communication lors de la 10 ème Journée de Recherche sur le e-marketing organisée par le Professeur Jean-François Lemoine à l'Université Paris 1 Panthéon Sorbonne, le 9 Septembre 2011.

${ }^{2}$ Oliviane Brodin, Maître de Conférences, HDR, I.A.E. de Lille, L.E.M. U.M.R. C.N.R.S. 8179, oliviane.brodin@iae.univ-lille1.fr

${ }^{3}$ Lise Magnier, Doctorante en Sciences de Gestion, I.A.E. de Lille, L.E.M. U.M.R. C.N.R.S. 8179, lise.magnier@ed.univ-lille1.fr
} 


\section{Introduction}

Cet article $^{4}$ présente la première étape du développement d'un index de mesure de l'exposition de soi dans les médias sociaux, un concept peu étudié en comportement du consommateur. Comprendre les spécificités des comportements des internautes est devenu un enjeu important pour le marketing comme pour d'autres disciplines telles les sciences politiques ou la sociologie. Les entreprises doivent adapter en permanence leurs pratiques relationnelles ou de communication à des internautes qui s'exposent, affichent leurs goûts, expriment leur opinion, présentent leurs réalisations, leurs relations ou leurs problèmes et racontent leurs expériences personnelles dans les médias sociaux, publiquement, dans des réseaux, des communautés ou entre amis. Ces derniers s'expriment en particulier sur leurs activités de consommation, avec un potentiel plus ou moins large et profond de transmission ou d'influence. L'essor massif des médias sociaux ${ }^{5}$ est aujourd'hui reconnu : trois Français sur quatre sont utilisateurs d'Internet et $78 \%$ utilisent les réseaux sociaux ${ }^{6}$. Si ces médias évoluent très rapidement, avec l'usage des chats et des blogs ${ }^{7}$, puis l'engouement pour les réseaux sociaux, leur utilisation n'est plus l'apanage des adolescents ${ }^{8}$. Dans ce contexte, il est important de mieux comprendre les comportements des internautes, leurs motivations et les rôles qu'ils jouent entre eux ou en relation avec les entreprises et les marques (fans, ambassadeurs de marque, leaders d'opinion et autres internautes influents). Le constat d'un internaute très ambivalent dans sa relation aux entreprises, participant enthousiaste (Divard, 2010), se surexposant spontanément en public (Tisseron, 2001), affichant ses commentaires, ses expériences, ses photos et ses amis et en même temps souvent attaché à la protection de sa vie privée (Joinson and Paine, 2007) demande à être approfondi.

Un concept intéressant comme source de compréhension de ces comportements des utilisateurs des médias sociaux, l'exposition de soi, semble sous-tendre bien des usages des médias sociaux. L'exposition de soi peut être définie comme une pratique sociale de «mise en visibilité de soi sur Internet pour laquelle le public est ratifié mais pas complètement identifié » (Granjon et Denouël, 2010). Sujet d'intérêt récent, l'exposition de soi n'est pas un terme consensuel; il est donc nécessaire d'évaluer sa pertinence par rapport à d'autres concepts à première vue très proches, comme le dévoilement de soi (ou " self disclosure »), l'extimité, l'exhibitionnisme, la visibilité médiatisée, ou la présentation de soi médiatisée. La nature du concept n'est pas clairement définie ou tient de diverses approches. L'approche psychologique d'une part, inclut des concepts tels le dévoilement de soi et les questions posées par la protection de la vie privée ou «privacy» (Joinson et Paine, 2007), le déplacement des frontières de l'intimité, le rapprochement avec le narcissisme (Lasch, 1979)

\footnotetext{
${ }^{4}$ Les auteurs remercient Doriane Beauvois, Doctorante à l'I.A.E. de Lille, pour sa participation à la réalisation des entretiens.

${ }^{5}$ Par médias sociaux, il faut entendre : "un groupe d'applications en ligne qui se fondent sur l'idéologie et la technologie du Web 2.0 et permettent la création et l'échange du contenu généré par les utilisateurs » (Kaplan et Haenlein, 2010).

${ }^{6}$ Selon l'Observatoire IFOP des réseaux sociaux, en Février 2011.

7162 millions de blogs sont recensés en Mai 2011 par Blogpulse, 32\% des internautes lisent des blogs (source : Pew Internet, Janvier 2011), 700 milliards de vidéos ont été visionnées sur YouTube en 2010 (source : YouTube, Janvier 2011), Twitter rassemble 175 millions de membres dans le monde en 2010 (source : Twitter, Janvier 2011), 35 millions de professionnels sont présents sur Viadeo (source : Viadeo, Janvier 2011), 5 milliards de photos sont publiées sur Flickr (source: Flickr, Janvier 2011) et Facebook compte près de 700 millions d'utilisateurs dans le monde, dont près de 22 millions en France (source : Social Bakers, Mai 2011).

${ }^{8}$ La tranche d'âge la plus représentée chez les utilisateurs de Facebook, par exemple, est celle des 25-34 ans (36\%) et les $45-54$ ans y sont aujourd'hui bien présents (11\%), même si les plus jeunes comptent pour une large part (10\% pour les $16-17$ ans et $13 \%$ pour les $13-15$ ans) (source : Social Bakers, Mai 2011).
} 
ou l'exhibitionnisme (Holbrook, 2001 ; Saenger et alii, 2009). L'approche sociologique ou psychosociologique d'autre part, englobe quant à elle des concepts tels que la présentation de soi (Goffman, 1973 ; Martinot, 2002) et la présentation de soi médiatisée (Thompson, 2005), l'injonction à la visibilité (Aubert et Haroche, 2011) ou à la recherche d'un soi véritable (Flahaut, 2006) dans un contexte d'individualisme expressif (Allard et Vandenberghe, 2003), voire à la publicisation et à la marchandisation de soi (Bauman, 2008) ou au besoin de reconnaissance (Honneth, 2000). Il semble donc nécessaire de clarifier la nature du concept d'exposition de soi et de préciser l'approche retenue : l'approche psychosociale de la construction de l'identité et du soi (Kaufmann, 2004; Martinot, 2002). Enfin, en comportement du consommateur, le concept est encore très peu étudié : les travaux de Saenger et alli (2009) sur l'exhibitionnisme, Brodin et Sevin (2010) sur les témoignages extimes, ou Ezan, Mallet et Rouen-Mallet (2011) sur les blogs d'adolescents, traitent toutefois du sujet.

Le niveau auquel nous étudions le concept doit être mieux défini, de même que sa nature multidimensionnelle ; en effet, si la tendance à s'exposer est fondamentale, présente dès le plus jeune âge (Tisseron, 2001) et si la tendance à se dévoiler est constitutive de toutes les interactions visant à créer des relations intimes (Erikson, 1966), la tendance à l'exposition de soi dans les médias sociaux semble née de normes sociales contemporaines, des pratiques de la télé-réalité (Mehl, 1996) et des spécificités de la communication médiatisée et des médias sociaux (Turkle, 1995 ; Boyd, 2008), telle l'absence du corps dans l'interaction (Walther, 1996).

Enfin, dans une perspective de développement d'un instrument de mesure, il peut-être utile de distinguer une démarche conceptuelle formative d'une démarche réflective, la première étant focalisée sur les « causes » ou éléments de définition qui contribuent à former le construit et la seconde s'intéressant à ses reflets ou à ses manifestations (Crié, 2005). Les manifestations de l'exposition de soi, ses formes et ses modalités, ont fait l'objet de quelques études (Cardon, 2008, 2009 ; Granjon et Denouël, 2010), mais les sources ou constituants de l'exposition de soi sont encore peu connus.

L'objectif de cette recherche est d'identifier les indicateurs de l'exposition de soi dans les médias sociaux afin d'en développer un index de mesure. Nous en présentons ici la première étape qui consiste à spécifier le contenu du construit et ses indicateurs. Les questions posées peuvent s'énoncer ainsi : peut-on penser l'exposition de soi dans les médias sociaux comme la conjonction de facteurs constitutifs et quels sont ceux qui sont à prendre en compte afin d'essayer de couvrir le champ du construit ? Existe-t-il des processus relationnels sous-jacents spécifiques ou distincts qui permettent de les considérer comme indépendants?

C'est tout d'abord une réflexion conceptuelle qui structure notre travail : il est important de justifier la nature formative des indicateurs identifiés et c'est l'objectif de la revue de littérature. Dans une première partie, l'exposition de soi et les concepts proches de la littérature psychologique, sociologique et du comportement du consommateur sont présentés. L'approche psychosociale qui sous-tend notre travail est également présentée et nous permet de mieux définir le concept, sa nature ainsi que le niveau auquel nous l'étudions. Ensuite, de manière à recenser suffisamment d'indicateurs pour couvrir la totalité du champ défini, la base de la littérature a été enrichie par une étude exploratoire, exposée dans une deuxième partie qui précise la méthodologie de l'étude menée. Une étude qualitative par entretiens individuels a donc été réalisée afin d'atteindre notre objectif exploratoire et de découvrir, de compléter ou de préciser, pour ceux qui sont repérés dans la littérature, les indicateurs du 
concept. Enfin, dans une troisième partie sont présentés les résultats de l'analyse qui permettent de proposer une structuration de ces indicateurs en vue des étapes suivantes de développement de l'index. Les limites et les perspectives envisagées sont finalement exposées.

\section{L'exposition de soi dans les médias sociaux}

Afin de définir et spécifier le concept d'exposition de soi dans les médias sociaux, les concepts voisins dans la littérature psychologique, sociologique, psychosociale et en comportement du consommateur sont présentés : le dévoilement de soi, l'extimité, l'exhibitionnisme, la visibilité et la présentation de soi. Leur diversité témoigne d'approches différentes et de la complexité du concept. Cette première démarche permet de définir la nature de l'exposition de soi, de justifier l'intérêt du niveau retenu pour l'étude de l'exposition de soi, celui des médias sociaux, et de mieux définir ce concept et le type de mesure à développer.

\subsection{L'exposition de soi et les concepts voisins}

\subsubsection{Le dévoilement de soi et la construction de relations interpersonnelles intimes}

Le dévoilement de soi (les révélations intimes) ou "self-disclosure " "fait référence à la tendance à discuter d'informations personnelles avec d'autres personnes » (Kito, 2005), à la propension à parler de soi à d'autres (Archer, 1980, p.183). Le dévoilement de soi fut initialement considéré comme un trait de personnalité stable ; l'accent fut mis ultérieurement sur le contexte social, le considérant alors comme une norme sociale culturelle, ou encore comme étant fortement associé à certains contextes relationnels (créés par exemple par les « openers », étudiés par Miller, Berg et Archer (1983)). Plusieurs traits de personnalité sont associés à des différences individuelles de dévoilement de soi : le contrôle expressif ou «self monitoring ", la conscience de soi, «self consciousness » ou « self awareness »; la timidité apparaît comme un frein au dévoilement, l'estime de soi, en revanche, favorise ces formes d'expression. Un certain nombre de motivations au dévoilement de soi ont été identifiées par Derlega et Grzelak (1979) : la recherche de validation ou approbation sociale, l'expressivité, la recherche de compréhension de soi ou «self clarification», le contrôle social et le développement relationnel. Le dévoilement de soi a été étudié dans le contexte du développement de relations interpersonnelles intimes ; une norme de réciprocité dans les révélations entre les interlocuteurs crée une intimité relationnelle graduellement plus profonde, plus large, plus durable, attribuée à des motifs positifs et base d'un engagement relationnel (Jacobs, Hyman et McQuitty, 2001). En s'exposant dans les médias sociaux, les internautes attendent des retours, mais ne recherchent pas nécessairement la création de relations interpersonnelles intimes. L'exposition de soi est médiatisée, se produisant dans une intimité distante, sans co-présence et non nécessairement réciproque, souvent dans une intimité de réseau ou de communauté ; ce sont les contextes médiatiques et relationnels qui permettent de les différencier. Le dévoilement de soi est donc essentiellement contextuel et particulier dans les communications sur Internet. L'examen des mesures existantes du dévoilement de soi montre que ce concept est généralement considéré comme multidimensionnel (Leung, 2002) et mesuré essentiellement sous un angle réflectif; le concept lui-même est sans doute à considérer comme l'une des formes que peut prendre 
l'exposition de soi. Un concept plus spécifique aux relations médiatisées a été exploré en psychanalyse et en sociologie : l'extimité.

\subsubsection{L'extimité : une forme de l'expressivisme contemporain}

L'extimité est une recherche de validation de soi par l'autre, comme le dévoilement de soi, mais est spécifique à des contextes médiatiques. Cette validation ne s'opère plus par la force de liens interpersonnels et le secret, mais par le nombre et la publicité, par l'étendue et non plus par l'intensité. L'extimité est une «tendance à l'exhibition de son intimité via différents médias », une « forme d'extériorisation de l'intimité » (Chapelain, 2007) dans une logique de "souci de soi » (Foucault, 1984), de travail sur soi dans la communication avec autrui (Kaufman, 2004); elle est apposée et non opposée à l'intimité. Pour Tisseron (2001), l'extimité désigne « le mouvement qui pousse tout un chacun à mettre en avant une partie de sa vie intime »; il utilise le terme dans un sens très différent de la notion lacanienne d'extime ${ }^{9}$. Pour Kaufmann (2001), « l'extériorisation est intrinsèquement un mouvement de fondation de soi » et non un rapport particulier de l'intime au public. Selon Tisseron (2001), c'est d'abord « un désir de trouver une confirmation dans le regard ou dans les réponses de l'autre à ce que je lui propose », caractéristique des émissions de télé-réalité ou de "l'intimité instrumentale », conséquence des relations personnalisées et éphémères des internautes (Chapelain, 2007) qui rappellent les transformations de l'intimité décrites par Giddens (1992). L'extimité est souvent étudiée sous l'angle de ses dispositifs : diarisme, blogs, télé-réalité, pages personnelles. Selon Chatelain (2007), le diarisme en ligne conduit à une présentation de soi maîtrisée due à la présence d'un lecteur actif et assouplit le pacte autobiographique qui confond habituellement en une même identité le narrateur, le personnage et l'auteur (Lejeune, 1975); cette maîtrise n'exclut pas les jeux identitaires fréquents sur Internet (Turkle, 1995). Le contexte médiatique et relationnel est également prépondérant dans la présentation de soi (Goffman, 1959), ce qui permet de différencier l'extimité de l'exhibitionnisme au sens psychologique. En effet, l'extimité suppose une prise de risque, une recherche de légitimité ou de reconnaissance, un désir d'inscrire l'expérience dans l'espace médiatique ; ceux-ci se traduisent par une adaptation de la présentation de soi au contexte social, médiatique et relationnel qui est ignorée dans la présentation de soi figée, ritualisée et provocante propre à l'exhibitionnisme. L'exposition de soi comme l'extimité sont des concepts qui se comprennent essentiellement selon une approche psychosociale ; l'extimité peut être tenue, selon la littérature, pour un constituant de l'exposition de soi médiatisée. Ses manifestations et modalités ont été explorées en comportement du consommateur pour proposer des typologies de formes et modalités de dévoilement public de soi dans des blogs créés par des consommateurs pour participer à un concours (Brodin et Sevin, 2010) et sur Facebook (Ben Mimoun, Garnier et Gentina, 2010).

\subsubsection{L'exhibitionnisme matérialiste : la culture contemporaine de la consommation comme véhicule de l'expression de soi}

Dans son acception psychologique, l'exhibitionnisme est défini comme une exposition de soi inappropriée mais auto-gratifiante (Blair et Lanyon, 1981) à différencier du sens qui lui est donné en comportement du consommateur. Selon Holbrook (2001), il caractérise la culture des consommateurs contemporains ou «l'utilisation par les consommateurs des produits

\footnotetext{
${ }^{9}$ L'extimité selon Lacan (2006) désigne « un extérieur logé au-dedans du sujet »; le symptôme relève d'un telle extimité.
} 
comme signaux pour véhiculer ou exprimer leur concept de soi véritable, désiré ou idéal aux autres ». La consommation comme moyen d'expression du soi constitue le fondement de travaux importants en comportement du consommateur avec le concept de soi étendu de Belk (1988) ou la distinction entre signification publique et privée des possessions (Richins, 1994). L'exhibitionnisme diffère de la consommation ostentatoire dans la mesure où ce n'est pas le statut social qui est affiché, mais l'identité du consommateur. Saenger et alii (2009) développent une échelle de mesure de l'exhibitionnisme dans les médias sociaux qu'ils définissent comme : «la tendance à communiquer sur ses activités de consommation (i.e. goûts, loisirs, activités de consommation) pour attirer l'attention sur soi et informer les autres sur qui on est pour la satisfaction personnelle que l'on peut retirer de cette publicité de soi »; le but est alors une recherche de gratification narcissique, une forme d'expression de soi pour soi. Saenger et alii (2009) privilégient une approche individuelle psychologique du concept : pour eux, le consommateur est, par nature, plus ou moins exhibitionniste. De plus, l'exhibitionnisme étudié par ces chercheurs est focalisé sur la consommation ; pourtant, même définies au sens large, les activités de consommation ne sont pas les seules expressions possibles de l'identité des internautes. Dans l'optique de Saenger et alii (2009), l'exhibitionnisme est un construit à comparer au leadership d'opinion, au «marketmavenisme », à l' "évangélisation ». Les consommateurs exhibitionnistes sont hypothétiquement matérialistes, narcissiques, extravertis et possèderaient une bonne confiance en soi, selon leur approche psychologique; ils ne précisent d'ailleurs pas les processus relationnels qui peuvent être mis en jeu car ils sont centrés sur l'individu. Nous partageons avec eux l'idée selon laquelle l'étude de l'exhibitionnisme (et de l'exposition de soi) peut contribuer aux recherches sur le bouche à oreille. L'exhibitionnisme de Saenger et alii (2009) apparaît comme une modalité possible de l'exposition de soi dans les médias sociaux, une manifestation de l'exposition de soi. Il peut prendre la forme de l'exposition de soi «trash» ou impudique étudiée par Cardon (2009) et Granjon et Denouël (2010) en sociologie ou celle de l'ostentation; la source en est alors plus une recherche d'attention ou de visibilité que de reconnaissance, de validation ou d'approbation.

\subsubsection{La visibilité : une injonction sociale contemporaine}

Selon Aubert et Haroche (2011), la visibilité est de l'ordre de l'injonction : « aujourd'hui, il faut rendre visible l'activité de chacun sous peine qu'elle ne soit pas prise en compte », au risque de l'objectivation et de la dissolution de soi (Sartre, 1943). Il ne s'agit pas seulement de sentiment d'existence, mais de réputation (reputatio signifiant " évaluation » en latin) et l'on assiste, selon Haroche, au retour de l'honneur, avec des pratiques telles le «personal branding " et le nettoyage de la réputation en ligne. Dans cette optique, la visibilité constitue un mode de contrôle social. Cardon (2010) soutient une vision différente : "les mises en scène de soi, de ses qualités, de ses compétences, accompagnent une volonté d'élargir l'espace de visibilité dans lequel chacun manifeste aux autres sa singularité pour la faire reconnaître ». D'une dichotomie privé-public on passerait à un continuum plus ou moins visible choisi par l'individu dans les médias sociaux. Voirol (2005a) distingue plusieurs visibilités : la visibilité formelle (de l'univers du travail), la visibilité pratique (arrangement qui permet l'ordre social), la visibilité médiatisée et la visibilité sociale (de groupes sociaux). Il définit la visibilité médiatisée comme «l'ensemble des opérations pratiques menées par les acteurs pour signifier ce qu'ils font au yeux d'autrui ». La distinction qu'il opère entre visibilité médiatisée et visibilité sociale permet de placer la lutte pour la visibilité et la reconnaissance sociale (Honneth, 2000, 2005) dans le registre de la dernière. Allant plus loin dans son analyse, Voirol (2005b) identifie une visibilité instrumentale, une «visibilité stratégique dont la finalité est de contraindre autrui à l'attention et de susciter son adhésion 
immédiate » qui utilise des techniques de recherche d'attention et de normalisation de la présentation de soi. Contrairement à l'inter-reconnaissance entre pairs (Allard et Vandenberghe, 2003), la visibilité instrumentale réduit la présentation de soi à un paraître superficiel, dans le sens d'une réduction aux capacités fonctionnelles; il s'agit alors d'une représentation de soi. Voirol rejoint sur ce point Bauman (2008) et le point de vue du contrôle social par l'observation ou la surveillance de soi et des autres (Foucault, 1975 ; Elias, 1991). La mise en visibilité de soi stéréotypée des sites de rencontres ou des réseaux sociaux professionnels illustre aisément la visibilité instrumentale. Comme pour l'exhibitionnisme, la présentation de soi est figée, mais elle est ici extrêmement adaptée. Les formes de la visibilité instrumentale dans les médias sociaux, les stratégies des internautes (l'e-réputation, le «personal branding», par exemple) sont à placer dans le registre des modalités de l'exposition de soi ; mais la recherche de reconnaissance et le désir d'attention peuvent être pensés comme des sources de l'exposition de soi dans les médias sociaux.

\subsubsection{L'exposition de soi : représentation de soi et mise en scène}

Les termes de visibilité médiatisée, d'exposition de soi ou de présentation de soi médiatisée sont utilisés indifféremment par certains auteurs. Cardon $(2010, p .11)$ s'attache à analyser les « nouvelles pratiques d'exposition de soi », « les mises en scène de soi, de ses qualités, de ses compétences ». Thompson $(2005$, p.71) définit la présentation de soi médiatisée comme une «forme intime de présentation de soi libérée des contraintes de la coprésence ». Granjon et Denouël (2010) donnent pour définition à l'exposition de soi les «pratiques de mise en visibilité de soi sur Internet ». La présentation médiatisée de soi est plutôt à associer à une approche psychosociale et aux mises en scène de soi, c'est un terme assez générique; la visibilité tient surtout de la norme sociale et des stratégies de publicisation de soi, et l'exposition de soi met l'accent sur certaines formes de l'exposition, celles du corps, dans certains travaux de Cardon (2009) et Granjon et Denouël (2010). Cardon identifie quatre formes d'exposition de soi corporelle imagée dans son enquête " Sociogeek » : l'exposition traditionnelle, la pudeur ou impudeur corporelle, l'exhibition et la provocation. Il propose par ailleurs une typologie des formes de visibilité (tout montrer/ tout voir; se voir caché ; se montrer caché et se cacher/ se voir) (Cardon, 2008). En comportement du consommateur, Ezan, Mallet et Rouen-Mallet (2011) se sont penchés sur la présentation de soi et les stratégies de mise en scène de soi des adolescents dans leurs blogs; ils ont identifié quatre types de blogs : les blogs de fans, de journaux intimes, participatifs et de prescription.

La pluralité des termes ainsi que la prépondérance des approches par les manifestations ou modalités pratiques de l'exposition de soi dans les médias sociaux justifient la nécessité de spécifier le concept dans notre recherche.

\subsection{La spécification du construit d'exposition de soi dans les médias sociaux}

\subsubsection{Une approche psychosociale de l'exposition de soi}

L'exposition de soi est essentiellement une activité volontaire, une pratique sociale. Se montrer au regard de l'autre est fondamental dans la construction de chaque individu, mais on peut observer des différences interindividuelles dans la tendance à s'exposer ou à passer inaperçu. Ces différences peuvent être engendrées par l'estime de soi ou encore par la recherche ou l'évitement de situations sociales évaluatives. L'hypermodernité et la culture 
individualiste expressive contemporaine peuvent exacerber certains traits comme le narcissisme (Lasch, 1979). Cependant, l'exposition de soi n'est pas un trait de personnalité stable : s'exposer, la manière de le faire, les contextes d'exposition de soi sont éminemment culturels et sociaux. L'approche retenue est une approche psychosociale. Selon cette approche, la présentation de soi, qui constitue la composante comportementale du soi, a pour but de structurer l'interaction afin d'obtenir le résultat souhaité (Martinot, 2002), en jouant un rôle qui fluctue en fonction de la situation sociale et du contexte d'interaction (Goffman, 1959). C'est cette approche relationnelle et psychosociale qui est privilégiée dans le développement de la mesure de l'exposition de soi dans les médias sociaux.

\subsubsection{Le niveau de la mesure : l'exposition de soi dans les médias sociaux}

Les médias conditionnant les interactions et les modalités de l'expression, étudier l'exposition de soi, non pas au plan général mais à celui des médias sociaux se justifie pleinement. La sociologie des usages explore d'ailleurs les pratiques spécifiques des usagers des technologies de l'information et de la communication (Denouël et Granjon, 2011). La présentation de soi médiatisée diffère de la présentation de soi en situation de co-présence (Thompson, 2005). Plusieurs études montrent que les communications sur Internet peuvent engendrer un dévoilement de soi plus important qu'en face à face, dans des conditions d'anonymat et en contexte "privé». Plus généralement, les communications médiatisées par ordinateur $(« C M C »)$ produisent des « interactions hyper-personnelles» plus intimes (Walther, 1996; Joinson, 2001 ; Dietz-Uhler et alii, 2005). Cette intimité est une intimité distante, sans coprésence et pas nécessairement réciproque, ce qui peut suffire à expliquer l'importance du dévoilement du corps et de l'affectif dans ces communications. D'autres explications de ces caractéristiques de la communication sur Internet sont également possibles : le brouillage des repères public-privé (par exemple le «clair-obscur» décrit par Cardon, 2008) et bien plus, l'exposition et la conformité à la norme du groupe, comme dans les chats (Dietz-Uhler et alii, 2005). L'exposition de soi est alors formatée par le média, ses fonctionnalités induisent l'intensité et les modalités de l'exposition (Stenger et Coutant, 2009). Les motivations peuvent en être diverses (recherche de visibilité professionnelle, désir de présenter des traces de soi, etc.), mais participent à la compréhension du concept; il est donc pertinent de mesurer l'exposition de soi au niveau des médias sociaux de manière générale. L'exposition de soi est une tendance individuelle contextuelle, c'est pourquoi il est préférable de distinguer l'exposition de soi de co-présence de l'exposition médiatisée ; il est alors justifié d'étudier le concept au niveau des médias sociaux où la culture de la révélation de soi est devenue une norme sociale.

\subsubsection{Une démarche formative}

Le concept d'exposition de soi a été mis en relation avec des facteurs individuels comme l'estime de soi et le narcissisme. En marketing, ses manifestations intéressent les praticiens, soit directement pour la compréhension des comportements tels ceux des ambassadeurs de marque ou des «market-mavens », soit pour mieux comprendre les paramètres de l'influence individuelle potentielle (par exemple, le nombre d'amis augmente avec l'exposition de soi sur Facebook, selon Cardon, 2009). De même que le dévoilement de soi, l'exposition de soi apparaît comme un concept multidimensionnel dont les indicateurs ne sont pas clairement identifiés. Deux voies peuvent être suivies (Crié, 2005) : soit une démarche réflective qui se focalise sur les manifestations ou les conséquences de l'exposition de soi (Ben Mimoun, Garnier et Gentina, 2010 ; Ezan, Mallet et Rouen-Mallet, 2011) - les indicateurs dérivent alors 
leur sens du concept, soit une démarche formative qui essaie d'en identifier les dimensions sources (Collier et Bienstock, 2009); la définition du concept est alors issue de la démarche. C'est cette seconde approche que nous avons retenue afin de définir l'exposition de soi et d'en développer une mesure ; il ne s'agit pas de calculer directement un score d'exposition de soi, mais de la mesurer à partir de ses catégories sources ou indicateurs « causaux » à identifier dans le contexte des médias sociaux (blogs, réseaux sociaux, chats, forums et communautés).

\section{Méthodologie}

\subsection{Les méthodes d'étude sur les pratiques d'exposition de soi dans les médias sociaux}

La plupart des études menées sur l'exposition de soi dans les médias sociaux (Cardon et Delaunay-Teterel, 2006 ; Cardon, 2009 ; Saenger et alii, 2009 ; Granjon et Denouël, 2010 ; Brodin et Sevin, 2010 ; Ben Mimoun, Garnier et Gentina, 2010 ; Ezan, Mallet et RouenMallet, 2011), s'intéressent aux pratiques d'exposition de soi, à leurs formes ou modalités et à la création de typologies des utilisateurs de blogs ou de réseaux sociaux basées sur les manifestations de l'exposition de soi et les stratégies de mise en scène de soi. Cardon et Delaunay-Teterel (2006) ou Cardon (2009) utilisent des enquêtes en ligne sur les pratiques et modalités d'épanchement de soi sur Internet et des entretiens biographiques sur les règles d'énonciation et de gestion de la relation avec le public des blogs. Granjon et Denouël (2010) mènent des entretiens semi-directifs et une analyse discursive des marques d'autoidentification produites sur Facebook. Brodin et Sevin (2010) réalisent une étude de cas d'opération de témoignages participatifs extimes sous forme de blogs et font une analyse du discours des contenus publiés par les participants afin d'explorer les rôles et rhétoriques des blogueurs. Ben Mimoun, Gentina et Garnier (2010) conduisent des entretiens d'utilisateurs de Facebook, dans une démarche interprétativiste et en proposent une analyse sémiotique : ils identifient quatre types d'utilisateurs: les authentiques, les artificiels, les extimes et les trompeurs. Enfin, Ezan, Mallet et Rouen-Mallet (2011) fondent leur étude sur la logique de découverte de Glaser et Strauss (1967) et observent des blogs d'adolescents durant trois mois; les grilles d'observation définies et les journaux de bord des auteurs sont ensuite analysés selon une analyse thématique et soumis à des experts; quatre types de blogs d'adolescents sont identifiés : le blog fan, journal intime, participatif et de prescription.

L'étude de Saenger et alii (2009) est la seule à avoir pour objectif de développer une échelle de mesure de l'exhibitionnisme comme tendance psychologique présentant des différences d'intensité interindividuelle. Leur démarche méthodologique est la suivante : une première liste d'items est générée à partir de la littérature et de focus groups ; elle est soumise ensuite à des experts. La phase suivante consistera à valider l'échelle.

Les études existantes traitent essentiellement des pratiques d'exposition de soi et sont souvent centrées sur certains médias (blogs, Facebook) ou sur des utilisateurs particuliers (les adolescents). Quant à l'étude de Saenger et alii (2009), elle suit une approche plus individualiste et psychologique que relationnelle et psychosociale. Les études les plus focalisées sur le sujet nous sont donc assez peu utiles pour identifier des indicateurs sources de l'exposition de soi dans les médias sociaux, à l'exception de celle de Saenger et alii (2009) 
qui établit un lien hypothétique avec l'estime de soi et le concept de soi, la satisfaction personnelle de se montrer en public et le narcissisme ${ }^{10}$.

\subsection{La méthodologie de l'étude}

Notre objectif est de recenser l'ensemble des indicateurs sources pour couvrir le champ de l'exposition de soi dans les médias sociaux (Collier et Bienstock, 2009) tout en demeurant dans le champ d'un nombre opérationnel d'indicateurs. Un certain nombre d'entre eux ont tout d'abord été recensés dans la littérature, cette recherche a ensuite été enrichie par des indicateurs issus d'une étude qualitative exploratoire, plus spécifiques à notre contexte d'étude. Ainsi, les indicateurs présentés dans les résultats sont soit de nouveaux indicateurs issus du terrain, soit des indicateurs déjà identifiés, mais qui sont alors précisés, déclinés et énoncés dans les termes des internautes. Il est irréaliste de penser atteindre cet objectif de recensement complet, mais l'étude exploratoire a pour but de s'en approcher.

Nous avons mené des entretiens individuels, choisis pour faciliter l'expression sur ce sujet personnel. Le guide d'entretien succinct et ouvert s'inspire des entretiens conceptuels décrits par Kvale (2007, p. 71) et développe les thématiques suivantes : - le sens et la représentation, pour l'interviewé, de son exposition dans les médias sociaux, la définition subjective qu'il donne de l'exposition de soi, - la problématique de l'exposition de soi en lien avec l'identité et les relations au public, les motifs et l'histoire personnelle de son exposition sur les médias sociaux et - les motifs ou fondements de la gestion de son identité et des contenus exposés dans ces médias. Les entretiens incluent les médias sociaux que sont : les réseaux sociaux personnels ou professionnels (de type Facebook, Viadeo, etc.), généralistes ou spécialisés, les blogs, les pages personnelles, les sites de rencontre, de partage de photos ou de vidéos, les jeux et les forums de discussion. Ces médias sociaux donnent la possibilité aux internautes de se présenter (profil, mur, identité civile, etc.) plus ou moins publiquement ou masqués, publier leurs créations (textes, photos, podcasts, vidéos, etc.), s'afficher ou se dévoiler aux autres, connus ou inconnus, échanger avec eux ou simplement voir ce qui est publié par d'autres. Les entretiens d'une durée moyenne de 53 minutes ont été enregistrés puis retranscrits intégralement.

L'échantillon comprend 18 internautes utilisateurs de médias sociaux divers et présentant des profils que nous avons cherché à diversifier en termes d'utilisation des médias sociaux et d'exposition de soi (l'un d'entre eux gère une cinquantaine d'adresses mails, est présent dans de nombreux réseaux sociaux généralistes et a créé une base de données de casting, une autre est très présente sur Facebook dans une période importante de sa vie, la naissance de son premier enfant (tableau 1 ci-dessous). La saturation a été atteinte par constat de la redondance des informations collectées.

\footnotetext{
${ }^{10}$ A notre connaissance, les 32 items initiaux de leur future échelle ne sont pas publiés à ce jour.
} 


\begin{tabular}{|c|c|c|c|c|c|}
\hline Prénom & Age & Sexe & Profession & Présence médias sociaux & $\begin{array}{l}\text { Contacts : (Amis) } \\
\text { Followers) }\end{array}$ \\
\hline Angélique & 23 & $\mathrm{~F}$ & Nourrice & Facebook*, MySpace, Blog, Msn, books de photo en ligne & Facebook : +200 \\
\hline Dorothée & 26 & $\mathrm{~F}$ & Enseignante & Viadeo*, Facebook, Blog 4LTrophy & $\begin{array}{l}\text { Facebook : } 20 \\
\text { Viadeo }: 170\end{array}$ \\
\hline Sandrine & 45 & $\mathrm{~F}$ & $\begin{array}{l}\text { Recherche d'emploi } \\
\text { (Ressources Humaines) }\end{array}$ & Forum (scoliose.org)*, Facebook, Viadeo, LinkedIn & Facebook : 34 \\
\hline Emma & 25 & $\mathrm{~F}$ & Chargée de recrutement & Facebook & Facebook : 209 \\
\hline Marion & 26 & $\mathrm{~F}$ & $\begin{array}{l}\text { Etudiante en Sciences de } \\
\text { l'Education }\end{array}$ & Facebook*, Msn, Copains d'avant & Facebook : +200 \\
\hline Julien & 24 & $\mathrm{H}$ & Ingénieur & Viadeo, Facebook, Msn, Copains d'Avant, OVS & Facebook : 30 \\
\hline Rachida & 23 & $\mathrm{~F}$ & Etudiante en commerce & Facebook & Facebook : 353 \\
\hline P. Alexis & 24 & $\mathrm{H}$ & Etudiant en Droit & Msn, Facebook & Facebook : 100 \\
\hline Leila & 22 & $\mathrm{~F}$ & Etudiante en Gestion & Msn, Facebook & Facebook : 211 \\
\hline Jérémy & 28 & $\mathrm{H}$ & Ingénieur & Viadeo*, Msn, LinkedIn, Copains d'Avant & Viadeo : 30 \\
\hline Paul & 22 & $\mathrm{H}$ & $\begin{array}{l}\text { Assistant- réalisateur/ } \\
\text { Photographe }\end{array}$ & $\begin{array}{l}\text { Facebook, Msn, FlickR, Meetic, Badoo, sites de jeux- } \\
\text { vidéo, casting }\end{array}$ & Facebook : +300 \\
\hline Laury & 23 & $\mathrm{~F}$ & $\begin{array}{l}\text { Assistante } \\
\text { communication }\end{array}$ & Msn, Viadeo, Facebook & Facebook : 100 \\
\hline Jean & 52 & $\mathrm{H}$ & Chef d'entreprise & Msn, Blog personnel, Blog professionnel, Facebook & Facebook : 30 \\
\hline Armand & 32 & $\mathrm{H}$ & Pianiste & $\begin{array}{l}\text { Twitter, } 2 \text { Facebook (personnel et professionnel), Site } \\
\text { Internet personnel, Blog }\end{array}$ & $\begin{array}{l}\text { Facebook } 1: 1500 \\
\text { Facebook } 2: 600 \\
\text { Twitter }: 235\end{array}$ \\
\hline Manon & 25 & $\mathrm{~F}$ & Responsable de projet & Facebook*, Msn, Adopteunmec & Facebook : 450 \\
\hline Clothilde & 51 & $\mathrm{~F}$ & $\begin{array}{l}\text { Chargée de } \\
\text { communication }\end{array}$ & Twitter*, Facebook (personnel et professionnel) & Facebook : 400 \\
\hline M. Antoine & 16 & $\mathrm{H}$ & Lycéen & Facebook*, Msn & Facebook : 360 \\
\hline Alcide & 20 & $\mathrm{H}$ & Etudiant en Droit & Forum jeu en ligne & Indéterminable \\
\hline
\end{tabular}

* Média le plus utilisé par le répondant

Tableau 1 : Profil des personnes interviewées

L'analyse a été réalisée selon un codage ouvert des énoncés produits dans les entretiens qui privilégie l'expression des interviewés. Sur la base de cette analyse, d'une observation complémentaire des contenus publiés (et dévoilés à l'interviewer durant l'entretien et $a$ posteriori) par les personnes interviewées et de mémos rédigés sur chacun des entretiens, les indicateurs sources de l'exposition de soi ont pu être identifiés, déclinés selon les expressions des interviewés, puis structurés en catégories plus formelles. Cette structuration a été réalisée selon les registres relationnels identifiés à partir du codage et par comparaison avec les catégories émanant de la littérature. 


\section{Résultats : les indicateurs constitutifs de l'exposition de soi dans les médias sociaux}

\subsection{Les indicateurs issus de la littérature et générés par l'étude qualitative}

\subsubsection{Les indicateurs émanant de la littérature}

Nous avons tout d'abord pris en compte les indicateurs émanant de la littérature et qui nous ont paru pertinents pour le développement de notre mesure. Plusieurs indicateurs ont été identifiés comme des indicateurs possibles de l'exposition de soi dans les médias sociaux : l'extimité, la validation de soi ou approbation de soi, la reconnaissance, l'expression d'un soi véritable, l'invention de soi, le développement de relations interpersonnelles médiatisées (intimes ou flexibles), la recherche d'attention, la visibilité instrumentale et l'obéissance à une injonction de visibilité. L'étude qualitative a confirmé qu'ils étaient présents chez les personnes interviewées et a permis de les décliner plus précisément selon les termes utilisés.

\subsubsection{Les indicateurs issus de l'étude qualitative}

L'analyse a permis de découvrir sept autres indicateurs plus spécifiques à l'exposition de soi dans les médias sociaux: l'officialisation (à la fois engagement et recherche de soutien), l'existence et la présence publiques, l'expression libérée du rôle social, la production quantitative de signes ou indicateurs de soi, l'affichage ou le prestige, l'influence (emprise ou être suivi), la rivalité (confrontation ou contestation).

- L'officialisation: le désir d'officialiser un statut, de s'installer dans un rôle social ou de s'engager publiquement est une recherche de validation sociale et de soutien, de fixation ou de définition de soi. Elle peut prendre la forme de l'affichage de son statut «marital» sur Facebook, d'un besoin de prise de parole dense ou d'affichage d'images de soi fort après un événement personnel important (maternité ou rupture, par exemple) ou encore d'un " coming out » ou d'une affirmation de soi comme membre d'un groupe social cherchant une visibilité. Les travaux d'Honneth (2000) sur la reconnaissance et ceux de Mehl (1996) sur la télévision de l'intimité analysent cette dimension plus généralement.

- L'existence et la présence publiques : rechercher une présence déployée dans l'espace des médias sociaux, pas nécessairement des traces durables mais prendre sa place, jouer un rôle reconnu par certains ou dans certains groupes est une dimension à rapprocher de la théorie de l'expression dans le domaine public de Sennett (1974, p. 15). Selon cet auteur, cette expression est motivée par un désir de s'authentifier comme acteur social, à différencier de l'expression d'un soi véritable, typique des relations de proximité ; elle serait en soi autogratifiante, créant un «élargissement» de soi à une dimension qui permet de sortir de soimême et d'exister à ses propres yeux par une existence publique. Merzeau (2010) indique que la présence numérique permet de déplacer l'angle de vision, de l'identité aux images, traces, profils laissés par les usagers.

- L'expression libérée du rôle social: le fait d'exprimer des pans secrets de soi, des problèmes intimes, de s'exprimer sans risques (à l'abri de l'entourage), de se montrer " décontrôlé », de s'épancher, de se raconter, constitue un motif d'exposition de soi qui n'était pas encore clairement identifié dans la littérature.

- La production quantitative de signes ou indicateurs de soi dans les médias sociaux: au service de la mesure de soi, elle permet de se quantifier soi-même afin de se situer, s'autoévaluer et se comparer aux autres ou leur donner les moyens de nous évaluer. C'est une démarche que l'on peut rapprocher de la «marchandisation de soi », selon les termes de 
Bauman (2008) ou Aubert et Haroche (2011), une amélioration de sa qualité selon des critères fonctionnels, que l'on peut juger très déshumanisante.

- L'affichage et le prestige : les résultats de l'étude identifient clairement cet indicateur diffus dans la littérature ; il s'agit de séduire, c'est-à-dire d'attirer l'attention du plus grand nombre et d'obtenir une adhésion immédiate. Une visibilité instrumentale est alors recherchée : elle consiste à parader, s'étaler, s'afficher. La recherche de prestige se présente comme une démarche ostentatoire (pas nécessairement matérialiste). L'internaute peut afficher une image idéalisée de lui-même, une représentation du bonheur familial ou de la beauté, du bien-être physique, par exemple. En termes de manifestations, les registres privilégiés dépendent sans doute du capital culturel et des valeurs prônées par l'individu comme par le public auquel il se montre.

- L'influence, l'emprise et être suivi: il s'agit non seulement de se comparer avantageusement aux autres, mais aussi d'avoir une emprise sur un certain nombre de personnes et de susciter l'envie ou d'être imité ; la référence à Girard (1972) est assez aisée.

- La rivalité (confrontation ou contestation): cet indicateur se manifeste dans les résultats de l'étude et n'était pas identifié spécifiquement dans la littérature sur l'exposition de soi. La rivalité apparaît comme un déploiement possible de la comparaison sociale; la confrontation ludique ou sérieuse est bien présente, par exemple dans les entretiens des adolescents qui rivalisent au sein de leur groupe de pairs. Les amateurs également cherchent parfois à rivaliser avec les experts, à les remettre en cause, à diminuer leur dépendance à leur égard ou à contester leur autorité, ce qui rejoint les analyses de Lasch (1979, p.23) au plan général et de Flichy (2010) sur les motivations des amateurs, « pro-am » ou connaisseurs. Les autres internautes, eux, défendent plutôt le droit à l'expression des témoins ordinaires, souvent sur le registre du vécu émotionnel.

Le tableau 2 ci-dessous présente les indicateurs de l'exposition de soi dans les médias sociaux issus de la revue de littérature et de l'étude exploratoire selon la distinction entre extimité et visibilité. 


\begin{tabular}{|c|c|c|c|c|}
\hline \multicolumn{2}{|c|}{$\begin{array}{l}\text { Indicateur de } \\
\text { l'exposition de } \\
\text { soi dans les } \\
\text { médias sociaux }\end{array}$} & $\begin{array}{l}\text { Origine dans } \\
\text { la littérature* }\end{array}$ & Catégorie issue de l'étude & Exemples de verbatims de l'étude \\
\hline \multicolumn{5}{|c|}{ Extimité } \\
\hline \multirow{3}{*}{ 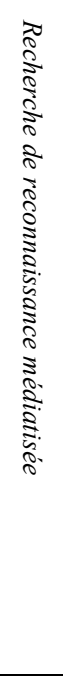 } & 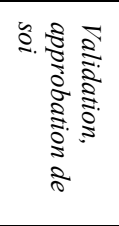 & Tisseron & $\begin{array}{l}\text { - être reconnu comme individu } \\
\text { singulier } \\
\text { - être validé par sa présence } \\
\text { dans certains médias sociaux } \\
\text { (innovativité, compétences, } \\
\text { créativité) }\end{array}$ & $\begin{array}{l}\text { "Je laisse les trucs que si c'est intéressant (une photo), ou si je } \\
\text { sais que ça peut plaire à quelqu'un parce par exemple le truc avec } \\
\text { les blue blockers c'est typiquement, c'est très culture américaine, } \\
\text { et je l'ai laissé parce que j'ai pas mal d'Américains dans mes } \\
\text { contacts et qu'ils voient que je connais un petit peu, que je suis pas } \\
\text { un Français qui connaît rien des Etats-Unis », Armand, L.378- } \\
381 \text {. }\end{array}$ \\
\hline & \multirow{2}{*}{ 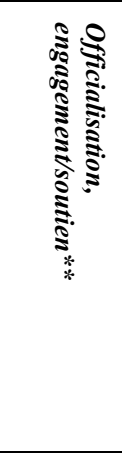 } & \multirow[t]{2}{*}{$\begin{array}{l}\text { (Honneth) } \\
\text { (Mehl) }\end{array}$} & $\begin{array}{l}\text { - être reconnu comme membre } \\
\text { d'un groupe social }\end{array}$ & $\begin{array}{l}\text { "Après j'ai une personnalité complètement différente donc qui est } \\
\text { celle du photographe dans laquelle je vais me vendre en qualité de } \\
\text { professionnel alors que je ne le suis absolument pas encore mais } \\
\text { où il faut que je montre une image de moi qui soit celle de } \\
\text { quelqu'un qui a confiance en lui, qui a de l'expérience, qui fait des } \\
\text { choses artistiques, etc. », Paul, L. 109-113. }\end{array}$ \\
\hline & & & $\begin{array}{l}\text { - officialiser un statut, } \\
\text { s'installer dans un rôle social, } \\
\text { - s'engager publiquement }\end{array}$ & $\begin{array}{l}\text { "En fait déjà je l'ai mis parce que mon copain est super jaloux } \\
\text { [...] je me suis dit que si par hasard un jour il tombe dessus, en } \\
\text { voyant le statut " en couple » il me prendrait pas la tête [...]. } \\
\text { C'était un peu pour me sauver quelque part d'une éventuelle } \\
\text { dispute", Laury L. 80-89. } \\
\text { "En ce moment je me remets au sport, c'est vrai j'ai tendance à le } \\
\text { dire pour aussi quelque part, indirectement, avoir des gens qui } \\
\text { derrière me disent "ah, c'est bien», Marion, L.143-144. }\end{array}$ \\
\hline \multirow{4}{*}{ 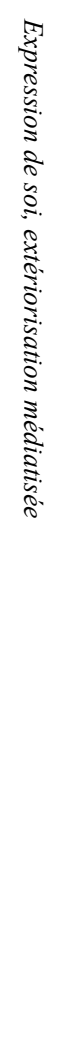 } & 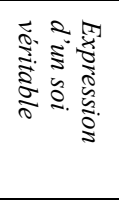 & $\begin{array}{l}\text { Allard et } \\
\text { Vandenberghe }\end{array}$ & $\begin{array}{l}\text { - exprimer un soi authentique, } \\
\text { sincère, véritable }\end{array}$ & $\begin{array}{l}\text { "Mais réellement c'est mon quotidien quoi !! Le quotidien que je } \\
\text { vis qu'il soit banal ou pas [...] et donc montrer ce que je suis en } \\
\text { train de faire et aussi parce que là ou je suis maintenant, enfin } \\
\text { transmettre à mes amis qui sont restés au bled comme quoi euh je } \\
\text { m'amuse et voila je vis ce que j'ai envie de vivre euh voilà ", } \\
\text { Rachida, L64-68.130-131. }\end{array}$ \\
\hline & 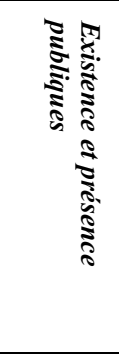 & (Sennett) & $\begin{array}{l}\text { - exister comme acteur social, } \\
\text { - élargir sa vie, ne pas rester } \\
\text { centré sur soi }\end{array}$ & $\begin{array}{l}\text { "D'autre part faire bouger et et améliorer le niveau de discussion, } \\
\text { là c'est spécial, c'est surtout par rapport à Twitter, [...], il y a } \\
\text { déjà des pages Facebook qui sont : " oh, regardez la jolie photo } \\
\text { que j'ai faite à la plage »,[...], moi je trouve que ce genre de chose } \\
\text { n'a aucun intérêt, or, sur Twitter je peux à la fois annoncer un } \\
\text { petit truc qu'on fait à Belle Ile et également leur dire " regardez, } \\
\text { au niveau international voilà ça ou ça qui se passe »,[...]. Mais il } \\
\text { me semble que ça me permet d'améliorer, d'avoir un esprit } \\
\text { d'ouverture, d'avoir un esprit, euh voilà autre que local, très [...]. } \\
\text { Voilà que c'est tout le contraire de l'exhibition, ,[...]presque, } \\
\text { quoi », Clothilde, L.64-74. }\end{array}$ \\
\hline & 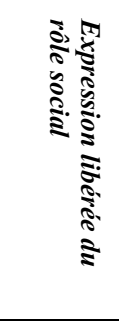 & & $\begin{array}{l}\text { - exprimer des pans secrets de } \\
\text { soi, des problèmes intimes } \\
\text { - s'exprimer sans risques (à } \\
\text { l'abri de l'entourage) } \\
\text { - se montrer « décontrôlé » } \\
\text { - s'épancher, se raconter }\end{array}$ & $\begin{array}{l}\text { "Mais je ne mets (sur les blogs) aucune info personnelle je ne } \\
\text { mets ni mon age ni mon adresse e-mail ni mon vrai nom, [...], et } \\
\text { voilà, même au boulot j'ai pas envie que les gens sachent que je } \\
\text { m'implique dans une cause politique", Clothilde L.427-438. "Je } \\
\text { peux m'exprimer de façon plus virulente on va dire, moins de } \\
\text { retenue ; par exemple, je vais reTwitter " au secours, là il faut vite } \\
\text { lâcher une bombe sur tel endroit» (en parlant des révolutions } \\
\text { arabes), jamais un truc que je vais dire avec un proche, d'abordy } \\
\text { a pas lieu ici (rire), et d'autre part ça me semblerait un peu } \\
\text { exagéré comme façon de m'exprimer ", Clothilde L.493-497. }\end{array}$ \\
\hline & 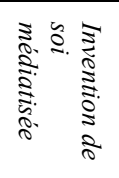 & $\begin{array}{l}\text { (Kaufmann), } \\
\text { Cardon }\end{array}$ & $\begin{array}{l}\text { - se tester, se construire } \\
\text { - se définir, s'essayer comme } \\
\text { - jouer des identités ou des } \\
\text { facettes de soi différentes, } \\
\text { multiples }\end{array}$ & $\begin{array}{l}\text { "Ouais, donc je disais, tu vas te mettre en avant moi, je suis } \\
\text { comme ça, comme ça, comme ça, mais oui, c'est un idéal qu'on } \\
\text { aimerait avoir sur le moment en fait. L'imaginaire de nous... », } \\
\text { Manon, L.172-174. }\end{array}$ \\
\hline \multirow[t]{2}{*}{ 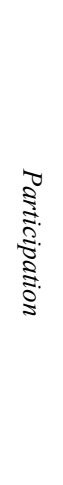 } & 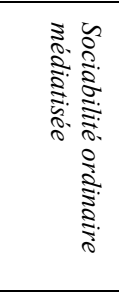 & (Walther) & $\begin{array}{l}\text { - se dévoiler, échanger pour } \\
\text { créer des liens interpersonnels } \\
\text { flexibles ou intimes } \\
\text { - informer son entourage des } \\
\text { événements quotidiens } \\
\text { - se présenter pour retrouver } \\
\text { d'anciennes connaissances } \\
\text { - nourrir ses relations } \\
\text { interpersonnelles proches }\end{array}$ & $\begin{array}{l}\text { "Parce que soit on est sur Facebook et je suis avec des amis, ils } \\
\text { connaissent ma vie ou parfois ils demandent si je suis à l'étranger } \\
\text { ou si je suis en voyage " mets tes photos ». Evidemment, je les } \\
\text { mets», Paul-Alexis, L.210-212. } \\
\text { "Comme y'avait pas mal de photos de soirée, de photos de repas, } \\
\text { d'événements, des photos d'un peu de tout quoi, ben d'une journée } \\
\text { dans une ville, ben c'était un moyen de partager ses photos et moi } \\
\text { je pouvais récupérer les photos des autres, etc. », Manon, L.77- } \\
80 \text {. }\end{array}$ \\
\hline & 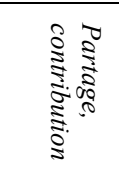 & Flichy & $\begin{array}{l}\text { - partager ses compétences, ses } \\
\text { connaissances, pédagogie, } \\
\text { apporter sa contribution à un } \\
\text { projet collectif }\end{array}$ & $\begin{array}{l}\text { "C'est un blog qui me sert à parler d'un sujet, notamment } \\
\text { l'assurance de prêt, comme moi, je suis courtier en assurances [...] } \\
\text { et donc euh, j'avais fait un blog sur l'assurance de prêt, c'est-à- } \\
\text { dire que je créais des articles, des petits billets ou tout ce qu'on } \\
\text { veut et puis j'essaye d'informer sur un sujet, en essayant de faire } \\
\text { un lien avec un site professionnel existant », Jean, L.44-51. }\end{array}$ \\
\hline
\end{tabular}




\begin{tabular}{|c|c|c|c|c|}
\hline \multicolumn{5}{|c|}{ Visibilité } \\
\hline \multirow[t]{3}{*}{ 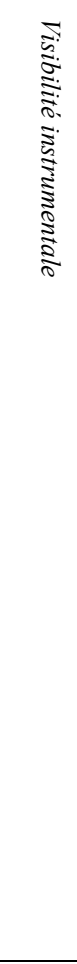 } & 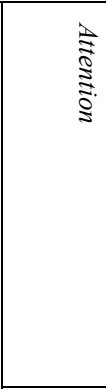 & $\begin{array}{l}\text { Voirol, } \\
\text { Thompson }\end{array}$ & $\begin{array}{l}\text { - attirer l'attention pour séduire } \\
\text { - construire son réseau social, } \\
\text { pouvoir contacter et être } \\
\text { contacté } \\
\text { - être bien référencé } \\
\text { - augmenter le trafic sur son } \\
\text { nom (site, blog...) } \\
\text { - construire sa notoriété, sa } \\
\text { popularité, sa réputation en } \\
\text { ligne }\end{array}$ & $\begin{array}{l}\text { "Je dirais que j'ai plusieurs objectifs avec Viadeo. } \\
\text { C'est être visible des recruteurs potentiels tout en étant en poste } \\
\text { actuellement. [...] avoir un profil visible... euh.... Deuxième point, } \\
\text { c'est développer un réseau professionnel en cas de licenciement ou } \\
\text { si je veux changer d'horizon. C'est une manière assez efficace de } \\
\text { contacter les personnes clés pour leur demander si ça bouge de } \\
\text { leur côté ou s'il y a des opportunités dans leur entreprise ou s'ils } \\
\text { ont entendu parler de postes sympas sur lesquels je pourrais me } \\
\text { positionner », Julien, L.117-121. } \\
\text { "J'essaie d'emprunter une communication qui s'apparente plus à } \\
\text { une communication de marque, plutôt qu'à une communication de } \\
\text { tout et n'importe quoi », Armand, L. 344-345. }\end{array}$ \\
\hline & 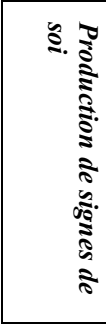 & (Bauman) & $\begin{array}{l}\text { - se mesurer/ être évalué } \\
\text { quantitativement } \\
\text { " googleiser »...) } \\
\text { - produire le plus d'indicateurs } \\
\text { possibles de soi } \\
\text { - s'améliorer selon des critères } \\
\text { fonctionnels } \\
\text { - construire son identité et sa } \\
\text { réputation en ligne } \\
\text { - se « marchandiser» }\end{array}$ & $\begin{array}{l}\text { «C'est pour ça que j'ai } 53 \text { adresses emails parce que selon le film } \\
\text { sur lequel je me trouve, j'ai besoin d'avoir une identité différente } \\
\text { [...] quant aux comptes Facebook, là c'est pareil, c'est lié à } \\
\text { plusieurs activités. En dehors de l'assistanat à la réalisation, j'ai } \\
\text { aussi une activité de photographe », Paul L.72-87. }\end{array}$ \\
\hline & 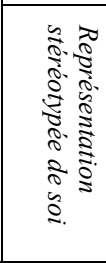 & $\begin{array}{l}\text { Aubert \& } \\
\text { Haroche }\end{array}$ & $\begin{array}{l}\text { - se soumettre à l'injonction de } \\
\text { visibilité } \\
\text { - imiter un modèle de } \\
\text { présentation de soi valorisé } \\
\text { dans un contexte défini } \\
\text { - adapter fortement sa } \\
\text { présentation à ce qui semble } \\
\text { attendu }\end{array}$ & $\begin{array}{l}\text { "Il va mettre des photos des férias assez ridicules, il met pas des } \\
\text { photos de férias pour partager avec ses amis, c'est pour montrer } \\
\text { qu'il est cool ", Dorothée L.367-370. } \\
\text { "Par exemple, j'avais mis une photo de moi où je me donnais } \\
\text { carrément un style. J'avais les cheveux attachés, mais un peu } \\
\text { lâches, un peu folle tu sais, un peu à la Brigitte Bardot, j'avais mis } \\
\text { des lunettes de star " mouche ", j'avais mon chat sur les jambes », } \\
\text { Marion, L.436-439. }\end{array}$ \\
\hline \multirow{3}{*}{ 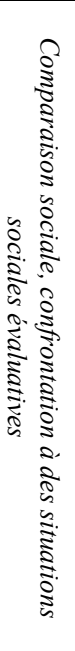 } & 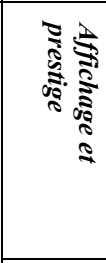 & $\begin{array}{l}\text { Saenger et al. } \\
\text { (exhibitionnis } \\
\text { me } \\
\text { matérialiste et } \\
\text { manifestation } \\
\text { de } \\
\text { l'exposition } \\
\text { de soi) }\end{array}$ & $\begin{array}{l}\text { - présenter une représentation } \\
\text { idéalisée de soi (bonheur } \\
\text { familial, beauté physique, etc.) } \\
\text { - parader, s'étaler, s'afficher }\end{array}$ & $\begin{array}{l}\text { "Quand j'ai mis les photos c'est vrai que je voulais montrer, me } \\
\text { montrer, je voulais montrer que je me sens bien que je voyage et je } \\
\text { visite des pays ou que je sors avec des amis, que je mène la belle } \\
\text { vie, c'est pas seulement les études quoi !!!! ", Leila, L.264-267. } \\
\text { "Tu sais, moi aussi j'ai mon âge pour moi, j'ai } 26 \text { ans, je suis une } \\
\text { jeune adulte qui a une image à contrôler, qui ne veut montrer que } \\
\text { les côtés positifs, les côtés... les côtés honorables, je sais pas si } \\
\text { c'est très correct de dire ça», Marion, L.328. }\end{array}$ \\
\hline & 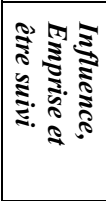 & & $\begin{array}{l}\text { - se comparer } \\
\text { - avoir une emprise sur les } \\
\text { autres, de l'influence } \\
\text { - susciter l'envie et être imité, } \\
\text { servir de modèle ou de } \\
\text { référence }\end{array}$ & $\begin{array}{l}\text { « Ben c'est une photo, ben les gens à mon avis, ils pensent ben } \\
\text { cette fille là elle s'amuse, elle a passé une bonne soirée et puis ben } \\
\text { elle a un grand sourire quoi, les mecs ils sont plutôt pas mal, ils } \\
\text { sont bien habillés, voilà quoi, ... peut-être qu'ils m'envient, donc } \\
\text { mais je sais pas, j'ai pas envie de la supprimer », Manon, L. 294- } \\
297 .\end{array}$ \\
\hline & 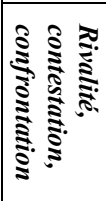 & $\begin{array}{l}\text { (Beck \& } \\
\text { Clark) } \\
\text { (Lasch) } \\
\text { (Flichy) }\end{array}$ & $\begin{array}{l}\text { - témoigner d'une expérience } \\
\text { vécue comme témoin ordinaire } \\
\text { - rivaliser avec son entourage, } \\
\text { dans son groupe } \\
\text { - contester des experts } \\
\text { (connaisseur, pro-am) }\end{array}$ & $\begin{array}{l}\text { «Dans certains cas, où je fais exprès de poster certaines choses } \\
\text { pour que certaines personnes les voient et que ça les fasse bien } \\
\text { chier », Armand, L.401-402. } \\
\text { "C'était entre guillemets un challenge qu'on s'était lancé entre } \\
\text { nous, pour savoir celle d'entre nous qui aurait le plus d'amis sur } \\
\text { Facebook», Emma, L.7-8. }\end{array}$ \\
\hline
\end{tabular}

- les références entre parenthèses renvoient à des auteurs qui ne font pas spécifiquement référence à l'exposition de soi dans les médias sociaux, mais que nous avons associés a posteriori à un indicateur issu de l'étude qualitative.

** les indicateurs issus de l'étude qualitative et non identifiés par la revue de littérature initiale sont mis en relief en caractères gras.

Tableau 2- Les indicateurs de l'exposition de soi dans les médias sociaux émanant de la littérature et de l'étude exploratoire. 


\subsection{L'exposition de soi dans les médias sociaux comme la conjonction de l'extimité et de la visibilité}

Le second résultat de notre étude tient dans la clarification des relations entre les indicateurs identifiés ou précisés dans l'étude. L'exposition de soi dans les médias sociaux apparaît comme la conjonction de deux indicateurs : l'extimité et la visibilité. Si les deux termes sont déjà présents dans la littérature, notre étude confirme la distinction entre les deux.

- L'extimité : reconnaissance, expression et participation dans un registre empathique. Le désir d'extimité apparaît dans notre étude comme une source importante de l'exposition de soi dans les médias sociaux. Notre étude précise que l'extimité peut être définie par trois indicateurs: la recherche de reconnaissance (la validation de soi et l'officialisation), l'expression de soi (d'un soi véritable, la recherche d'une existence publique, une expression libérée de la présentation du rôle social et l'invention de soi) et la participation (la sociabilité ordinaire et le partage ou contribution). L'extimité est ainsi définie de façon plus complète : la recherche d'une existence publique et l'expression libérée ne sont pas des aspects mis en relief dans la littérature. L'inclusion de la participation à ce niveau se justifie conceptuellement par le registre empathique commun à l'ensemble de ces dimensions : l'extimité se présente comme essentiellement relationnelle. Se focaliser sur l'individualisme expressif, la sensibilité à soi ou le narcissisme occulte ce registre empathique, de contact et de reconnaissance réciproques. Ce qui unit les analyses, aussi bien chez les psychanalystes et les sociologues que dans l'étude des échanges communicationnels, c'est le mécanisme commun à la présence et à l'expressivité : il s'agit d'être soi par l'autre et avec l'autre, pour être identifié, exister aux yeux des autres, être accepté, dans une relation à la fois dynamique et réciproque. Tisseron (2010, p. 54) qualifie d'ailleurs l'extimité ainsi : "dans une interaction empathique complète, l'autre nous révèle à nous-même, et nous nous révélons à lui »; c'est un processus de reconnaissance qui est en œuvre, une forme d' " inter-reconnaissance entre pairs ", selon Allard et Vanderberghe (2003). Elle permet d'identifier la personne et n'est donc pas confondue avec la reconnaissance sociale (Honneth, 2000).

- La visibilité : visibilité instrumentale et comparaison, confrontation dans un registre de situations sociales évaluatives. La seconde source de l'exposition de soi qui apparaît dans nos résultats est la recherche de visibilité, présente dans la littérature ; elle se distingue clairement de l'extimité et peut être considérée comme appartenant essentiellement au domaine de la désirabilité et du management de l'impression sociale. Elle est constituée, selon notre étude, de deux aspects : la visibilité instrumentale (la recherche d'attention, la production de signes de soi et la représentation stéréotypée de soi) et la comparaison sociale (l'affichage et le prestige, l'influence, l'emprise ou le désir d'être suivi et la rivalité, confrontation ou contestation). La visibilité consiste à attirer l'attention, à s'afficher ou à se mettre en scène, faire sa publicité ou se starifier, se mettre en valeur, parader et produire ou faire étalage, en permanence, d'une quantité de signes. Le premier pôle, la visibilité instrumentale, réduit l'autre à une position de spectateur passif et celui qui s'expose le fait dans une représentation de soi stéréotypée, conformiste, contrôlée (même dans le "décontrôle" culturellement valorisé dans certaines situations) et très adaptée : c'est une représentation fictive de soi qui est mise en avant par la production permanente de signes quantifiés de soi. Le registre identifié est celui de la séduction et du conformisme. Il est question d'impressionner, occuper la plus grande place possible dans l'espace et dans l'esprit du public par une adhésion immédiate obtenue en incarnant un modèle emprunté qui exige des sacrifices ; la visibilité instrumentale est de l'ordre de l'ostentation au sens large. Le second pôle de la visibilité est la comparaison ou la confrontation dans des situations sociales évaluatives. Dans ce cas, ce sont 
plutôt la compétition ou l'emprise qui prévalent avec la recherche de prestige, d'influence ou de rivalité : le regard nécessaire est d'abord celui du rival devant le public; les pratiques associées de construction de la réputation peuvent être celles des joutes ou encore celles des « pro-am » qui contestent l'autorité des experts.

\section{Discussion}

\subsection{Apports et limites de l'étude}

L'apport conceptuel de notre étude se situe dans la clarification du concept d'exposition de soi dans les médias sociaux. Cette étude exploratoire permet d'identifier deux sources constitutives du concept: l'extimité et la visibilité, ainsi que leurs constituants respectifs. L'extimité est donc composée de la reconnaissance, de l'expression et de la participation, et la visibilité est composée de la visibilité instrumentale et de la comparaison sociale. Les autres concepts associés, dans la littérature, à l'exposition de soi seraient donc à considérer plutôt comme des formes ou des manifestations de l'exposition de soi (l'exhibitionnisme matérialiste, le dévoilement de soi, par exemple). Nous avons identifié deux registres qui autorisent à considérer que l'extimité et la visibilité sont conceptuellement distinctes : un registre relationnel empathique pour l'extimité et un registre de comparaison ou d'évaluation sociale pour la visibilité. L'exposition de soi dans les médias sociaux se définit ici comme un construit de nature relationnelle et contextuelle, ce qui justifie le développement d'une mesure spécifique à ce niveau. L'approche psychosociale suivie met peut-être l'accent sur certains déterminants au détriment d'autres indicateurs. A ce stade, nous n'avons pas pris en compte les « coûts » ou les risques associés à l'exposition de soi, qui sont plus larges que la seule protection de la vie privée (Salerno, 2001). Enfin, bien que l'intérêt d'une approche formative soit reconnu, les limites de cette démarche ne sont pas à ignorer. Par exemple, certains indicateurs vus ici comme formatifs pourraient être conceptualisés par d'autres comme réflectifs.

L'apport méthodologique de cette recherche tient dans la création d'indicateurs issus de la littérature et du terrain qui permettra de développer un index pour une mesure globale de l'exposition de soi dans les médias sociaux. Dans cette perspective, les limites de l'étude doivent être énoncées : si les derniers entretiens ont apporté peu d'informations nouvelles, la diversité de l'échantillon pourrait être améliorée pour considérer que l'on a véritablement atteint la saturation sémantique (Glaser et Strauss, 1967); des cas plus extrêmes ne sont pas inclus, comme l'interview d'une personne à très forte notoriété ou des personnes recherchant l'invisibilité dans les médias sociaux, en contrepoint.

$\mathrm{Au}$ plan managérial, cette clarification du concept peut être utile à la compréhension des comportements des internautes dans les médias sociaux: quelles sont les motivations des internautes intéressés par des services de "personal branding", de gestion de l'identité numérique, par des outils permettant de créer sa carte de visite numérique ou de corriger son image dans les médias sociaux? Quelles sont les motivations de ceux qui inondent les sites de commentaires parfois acides et font du «trolling » en prenant pour cibles les médias et les marques ? Comment comprendre que les internautes acceptent de se faire les porte-parole de certaines marques gratuitement et réclament une rétribution pour leurs contributions par ailleurs ? Comment les activités de sociabilité deviennent-elles sources de recommandations marketing et de prescription de consommation (Stenger et Coutant, 2009) ? Cette étude montre que les contextes relationnels sont essentiels pour comprendre ces comportements et 
présente des éléments qui peuvent être intéressants pour mieux identifier les motivations des «market-mavens », leaders d'opinion et "e-fluents» dans les médias sociaux ainsi que les fondements et processus de leur influence, différents, dans certains cas, de l'expertise et de la crédibilité.

\section{2. $\quad$ Perspectives de recherche}

La première piste ouverte par cette étude consiste à poursuivre le développement d'un index de mesure de l'exposition de soi dans les médias sociaux. Une liste des indicateurs définis lors de cette première phase est à soumettre à des experts, afin de s'assurer, en particulier, de leur complétude apparente, la validité de contenu étant essentielle dans une démarche formative. Il nous faut sans doute au préalable compléter notre analyse sur la recherche d'invisibilité et le couple exhibitionnisme-voyeurisme. Seul le pan de l'exhibitionnisme a été exploré dans les résultats présentés ici. L'étude de la régulation du dévoilement de soi dans les médias sociaux, qui dépasse le concept de protection de la vie privée, est amorcée dans certaines études sur les stratégies des adolescents dans les réseaux sociaux (Tufekci, 2008) et pourrait être approfondie avec une recherche sur les risques perçus à l'exposition de soi dans les médias sociaux. Il faut également identifier des effets, des conséquences à mettre en relation avec les causes du construit qui nous intéresse (on peut penser au bouche à oreille narratif ou d'expérience personnelle, aux rôles de "market-mavens ", de porte-parole ou ambassadeurs de marques, aux modèles de rôles, aux "taste-makers » et aux exhibitionnistes au sens de Saenger et alii (2009) ou encore à la participation à des opérations de communication coproduites telles les castings clients). En relation avec l'exposition de soi, plusieurs variables peuvent être à considérer, comme l'estime de soi, la conscience de soi ("self awareness » et "self consciousness ») qui peuvent différencier la présence dans les médias sociaux de l'exposition de soi. L'auto-contrôle expressif ou "self monitoring » (Snyder, 1974) pourrait intervenir différemment dans l'extimité et la visibilité. La désirabilité sociale et le conformisme peuvent également diriger ces motivations avec une intensité variable. Le modèle conceptuel reste à préciser et à tester.

Une autre manière de traiter l'exposition de soi consiste à distinguer la motivation à traiter l'information sur soi, pour soi (l'extimité) ou à observer plutôt la motivation à considérer l'impression produite et à l'influencer (management de l'impression et visibilité, pour l'exposition de soi). Pour la première, Martinot (2008) distingue trois grandes motivations au traitement de l'information sur soi : l'auto-valorisation (ou «self-enhancement", avec la recherche de stabilité et l'attention aux feedbacks sociaux), l'auto-évaluation («selfassessment » ou recherche de vérité sur soi, obligation éthique de rechercher sa vraie nature, pour soi) et la consistance (ou "self-verification », la connaissance de soi tel que l'on est (Snyder, 1983)). Le fait de chercher à se définir, à se fixer ou à s'affirmer dans un nouveau rôle social (auto-valorisation), ou bien tenter de se libérer de son rôle social pour s'exprimer plus pleinement, des motivations que nous avons pu identifier lors de notre étude, sont de l'ordre de ces motivations à traiter l'information sur soi. La seconde, le management de l'impression (Schlenker, 1980), concerne le résultat que l'on cherche à obtenir sur la perception de soi par l'autre, par l'influence, les tentatives pour impressionner ou les stratégies pour "se faire bien voir» et susciter la compassion. Il s'agit d'une forme de présentation de soi que l'on peut penser uniquement instrumentale ; l'exposition de soi, dans sa dimension de visibilité, serait une forme affirmative du management de l'impression (la recherche d'invisibilité pouvant être interprétée comme une forme défensive, d'évitement de situations sociales évaluatives). Mais le management de l'impression distingue également 
deux voies : instrumentale et expressive, elle paraît donc plus englobante, incluant des stratégies suscitant l'empathie, la séduction et l'intimidation. Il est cependant préférable de les distinguer sur la base de leurs objectifs fondamentaux, et si le renversement de registre de l'empathie à la rivalité entre expressivité (extimité) et instrumentalisation (visibilité) n'est plus aussi net selon l'approche par le management de l'impression, c'est que, dans ce cas, susciter l'empathie est instrumental et non réciproque : l'expressivité du management de l'impression est différente dans son but et plus étroite que l'extimité.

En conclusion, le concept d'identité relationnelle nous paraît enrichissant pour comprendre l'exposition de soi. Selon l'hypothèse de Flahaut (2006, p. 258), l'identité narrative (Ricoeur, 1985) ne se suffit pas à elle même et doit être pensée avec l'identité relationnelle. C'est une critique de l'approche individualiste soutenant la thèse de la consistance de soi et de la possibilité d'expression d'un soi véritable qui provient d'une conception du soi comme " substance ${ }^{11}$ ou noyau. Selon Flahaut (2006), la recherche d'un soi véritable est souvent confondue avec le sentiment d'exister, celui-ci n'exigeant pas de conscience de soi forte. La notion d'identité relationnelle implique que les relations interviennent en amont de la personne, dans sa constitution même (Flahaut, 2006, p. 261). Les connaissances actuelles montrent qu'exister nécessite de participer à ce qui n'est pas soi, se sentir dans l'interdépendance; cette relation à l'autre n'est pas de nature morale, mais constitutive de notre être. Flahaut oppose le sentiment d'existence (se sentir bien, en confiance, dans un processus de transmission, comme dans la conversation pour laquelle la " self consciousness » nuit) au désir d'impressionner (coller à une image). L'exposition de soi dans les médias sociaux, du moins l'extimité, selon ce point de vue, serait avant tout relationnelle et non expressiviste; plus largement, l'identité relationnelle, permettrait de comprendre la présence et l'activité dans les médias sociaux, avec les comportements de relais, de participation à des mouvances (politiques, éthiques, etc., que l'on trouve par exemple dans les motivations des acteurs de Twitter) qui offrent à l'individu la possibilité d'élargir ce sentiment d'existence.

\section{Bibliographie}

Allard L. et Vandenberghe F. (2003), « Express Yourself! Les pages perso entre légitimation techno-politique de l'individualisme expressif et authenticité réflexive peer-to-peer », Réseaux, No. 117, p. 191-219.

Archer J.L. (1980), "Self-disclosure », in D. Wegner et R. Vallacher (coord.), The Self in Social Psychology, Londres, Oxford University, p. 183-204.

Aubert N. et Haroche C. (2011), «Les tyrannies de la visibilité », Paris, Eres.

Bauman Z. (2008), "S'acheter une vie », Nîmes, Editions Jacqueline Chambon.

Belk R.W. (1988), "Possessions and the extended self», Journal of Consumer Research, Vol. 15, No. 2, p. 139-168.

Ben Mimoun M.S., Garnier M. et Gentina E. (2010), « Identité, extimité et marque sur Facebook : Une approche "Webnographique »", Actes du 15ème colloque de l'Association Information et Management, La Rochelle, Mai.

Blair C.D. et Lanyon R.I. (1981), «Exhibitionism: Etiology and treatment », Psychological Bulletin, No. 89, p. 439-463.

Boyd D. (2008), « Facebook's privacy trainwreck: exposure, invasion, and social convergence», Convergence, Vol. 14, No. 1, p. 13-20.

\footnotetext{
${ }^{11}$ Cette conviction a été forgée par la philosophie antique et le christianisme ; la théorie de la réalisation de soi, typiquement individualiste, sous-tend les écrits de Beck ou Giddens que critique Flahaut.
} 
Brodin O. et Sevin E. (2010), «Les règles de la coproduction de témoignages extimes de consommateurs dans une opération de communication participative : le cas de l'opération Ford S-Max », 5èmes Journées Internationales sur la Communication Marketing, Nancy, 2526 Mars.

Cardon D. (2008), «Le design de la visibilité. Un essai de cartographie du web 2.0 », Réseaux, Vol. 26, No. 152, p. 93-137.

Cardon D. (2009), "Sociogeek, identité numérique et réseaux sociaux », Paris, Fyp.

Cardon D. (2010), «La Démocratie Internet : Promesses et limites », Paris, Seuil.

Cardon D. et Delaunay-Téterel H. (2006), "La production de soi comme technique relationnelle. Un essai de typologie des blogs par leurs publics », Réseaux, Vol. 24, No. 138, p. 15-71.

Cauquelin A. (2003), «L'exposition de soi - Du journal intime aux webcams », Paris, Eshel.

Chapelain B. (2007), «L'intimité à l'écran : Une écriture de l'extimité ? », in P. Lardellier et P. Ricaud (coord.), Le réseau pensant: Pour comprendre la société numérique, Dijon, Éditions universitaires de Dijon.

Collier J.E. et Bienstock C.C. (2009), « Model misspecification: contrasting formative and reflective indicators for a model of e-service quality », Journal of Marketing Theory and Practice, Vol. 17, No. 3, p. 283-293.

Crié D. (2005), «De l'usage des modèles de mesures réflectifs ou formatifs dans les modèles d'équations structurelles ", Recherche et Applications en Marketing, Vol. 20, No. 2, p. 5-27.

Denouël J. et Granjon F. (2011), accessible à l'adresse URL suivante : http://www.internetactu.net/2011/06/15/julie-denouel-et-fabien-granjon-les-usages-enquestion/?utm_source=feedburner\&utm_medium $=$ feed\&utm_campaign $=$ Feed:+internetactu/b cmJ+(InternetActu.net).

Derlega V.J. et Grzelak A.L. (1979), « Appropriate self-disclosure », in Chelune G.J. (coord.), Self-disclosure: origins, patterns, and implications of openness in interpersonal relationships, San Francisco, Jossey-Bass.

Dietz-Uhler B., Bishop-Clark C. et Howard E. (2005), « Formation of and adherence to a selfdisclosure norm in an online chat », CyberPsychology and Behavior, No. 8, p. 114-120.

Divard R. (2010), «Le marketing participatif », Paris, Dunod.

Elias N. (1991), «La société des individus », Paris, Fayard.

Erikson E. (1966), "Enfance et société », Neuchâtel, Delachaux et Niestlé.

Ezan P., Mallet S. et Rouen-Mallet C. (2011), « Je blogue, donc je suis : les stratégies de mise en scène de soi des adolescents ", 10èmes Journées Normandes de Recherche sur la Consommation, IAE de Rouen, 17 Mars 2011.

Flahaut F. (2006), «Be Yourself», Paris, Mille et Une Nuits.

Flichy P. (2010), «La sacre de l'amateur », Paris, Seuil.

Flick U. (2009), « An introduction to qualitative research», Londres, Sage Publications Ltd. 4ème edition.

Foucault M. (1975), "Surveiller et punir », Paris, Gallimard.

Foucault M. (1984), «Histoire de la sexualité. Tome 3 : le souci de soi », Paris, Gallimard.

Giddens A. (1992), "La transformation de l'intimité- Sexualité, amour et érotisme dans les sociétés modernes », Paris, Le Rouergue/ Chambon.

Girard R. (1972), «La violence et le sacré », Paris, Hachette Littératures.

Glaser B.G. et Strauss A.L. (1967), "The discovery of grounded theory. Strategies for qualitative research », Chicago, Aldine Publishing Company.

Goffman E. (1959), «La Mise en scène de la vie quotidienne », Paris, Éditions de Minuit (édition de 1973).

Granjon F. et Denouël J. (2010), «Exposition de soi et reconnaissance de singularités subjectives sur les sites de réseaux sociaux », Sociologies, Vol. 1, No. 1, p. 25-43. 
Holbrook M.B. (2001), « The millennial consumer in the texts of our times: exhibitionism », Journal of Macromarketing, Vol. 21, No. 1, p. 81-95.

Honneth A. (2000), «La Lutte pour la reconnaissance », Paris, Cerf.

Honneth A. (2005), «Invisibilité : sur l'épistémologie de la reconnaissance», Réseaux, No. 129-130, p. 39-57.

Jacobs R.S., Hyman M.R et McQuitty S. (2001), « Exchange-specific disclosure, social disclosure, and personal selling », Journal of Marketing Theory and Practice, Vol. 9, No. 1, p. 48-62.

Joinson A.N. et Paine C.B. (2007), " Self-Disclosure, Privacy and the Internet ", in A.N. Joinson, K.Y.A. McKenna, T. Postmes and U.D. Reips (coord.), Oxford Handbook of Internet Psychology, Oxford, Oxford University Press.

Kaplan A.M. et Haenlein M. (2010), " Users of the world, unite! The challenges and opportunities of social media », Business Horizons, Vol. 53, No. 1, p. 59-68.

Kaufmann J.C. (2001), «Ego. Pour une sociologie de l'individu », Paris, Nathan.

Kaufmann J.C. (2004), «L'invention de soi : Une théorie de l'identité », Paris, Armand Colin.

Kito M. (2005), « Self-disclosure in romantic relationships and friendships among American and Japanese college students ", The Journal of Social Psychology, Vol. 142, No. 2, p.127140.

Kvale S. (2007), « Doing interviews », Londres, Sage Publications.

Lacan J. (2006), « D’un Autre à l'autre », Paris, Seuil.

Lasch C. (1979), «La culture du narcissisme- La vie américaine à un âge de déclin des espérances », Paris, Flammarion.

Lejeune P. (1975), "Le Pacte autobiographique », Paris, Seuil.

Leung L. (2002), " Loneliness, Self-Disclosure, and ICQ ("I Seek You") Use », CyberPsychology and Behavior, Vol. 5, No. 3, p. 241-251.

Martinot D. (2002), «Le soi. Les approches psycho-sociales», Grenoble, PUG.

Martinot D. (2008), "Le soi, les autres et la société », Grenoble, PUG.

Mehl D. (1996), «La télévision de l'intimité », Paris, Seuil.

Merzeau L. (2010), «Présence numérique : de la gestion d'une identité à l'exercice d'une liberté », Revue DocSI, Vol. 47, No. 1, p. 28-69.

Miller L.C., Berg J.H. et Archer R.L. (1983), « Openers: Individuals who elicit intimate selfdisclosure ", Journal of Personality and Social Psychology, Vol. 44, No. 6, p. 1234-1244.

Richins M.L. (1994), "Valuing things: the public and private meanings of possessions ", Journal of Consumer Research, Vol. 4, No. 3, p. 504-521.

Ricoeur P. (1985), « Temps et récit, Tome 3 : temps raconté », Paris, Seuil.

Saenger C., Thomas V., Wiggins Johnson J. et Jewell R. (2009), « Exposing consumer exhibitionists: the development and validation of the consumer exhibition scale ", Advances in Consumer Research, Vol. 37, p. 895-896.

Salerno F. (2001), "Webmarketing : obtenir la confiance du consommateur », Revue Française de Gestion, No. 134, p. 66-80.

Sartre J.P. (1943), "L'être et le néant », Paris, Gallimard.

Schlenker B.R. (1980), "Impression management: the self-concept, social identity, and interpersonal relations », Monterey, Brooks/Cole.

Sennett R. (1974), «Les tyrannies de l'intimité », Paris, Seuil.

Stenger T. et Coutant A. (2009), «La prescription ordinaire de la consommation sur les réseaux socionumériques : De la sociabilité en ligne à la consommation ? ", 14e Journées de Recherche en Marketing de Bourgogne, Dijon, p. 40-60.

Snyder M. (1974), " Self-monitoring of expressive behavior », Journal of Personality and Social Psychology, No. 30, p. 526-537.

Thompson J.B. (2005), « La nouvelle visibilité », Réseaux, Vol. 23, No. 129-130, p. 59-87. 
Tisseron S. (2001), "L L'intimité surexposée », Paris, Hachette.

Tisseron S. (2010), «L'empathie au coeur du jeu social », Paris, Albin Michel.

Tufecki Z. (2008), "Can you see me now? Audience and disclosure regulation in online social network sites », Bulletin of Science, Technology \& Society, Vol. 28, No. 1, p. 20-36.

Turkle S. (1995), "Life on the screen-Identity in the age of the Internet », New York, Simon \& Schuster.

Voirol O. (2005a), «Visibilité et invisibilité : une introduction », Réseaux, Vol. 23, No. 129130, p. 9-36.

Voirol O. (2005b), «Les luttes pour la visibilité. Esquisse d'une problématique », Réseaux, Vol. 23, No. 129-130, p. 89-121.

Walther J.B. (1996), « Computer-mediated communication. Impersonal, interpersonal, and hyperpersonal interaction », Communication Research, Vol. 23, No. 1, p. 3-43. 